(RESEARCh ARTicLE)

\title{
A Comparative study on the cost effectiveness of intervention of noncommunicable disease in south Darfur 2017
}

\author{
Yousif Adam Hussien Noreldin ${ }^{1}$ and Ekram Adam Eldoom 2, * \\ ${ }^{1}$ RH/GBV Program Analyst Team Leader UNFPA South and East Darfur states 0912321229. \\ ${ }^{2}$ Faculty of Public Health, University of Alzaeim Alazhari, Sudan.
}

Magna Scientia Advanced Biology and Pharmacy, 2021, 02(01), 019-025

Publication history: Received on 26 February 2021; revised on 30 March 2021; accepted on 03 April 2021

Article DOI: https://doi.org/10.30574/msabp.2021.2.1.0012

\begin{abstract}
Background: This study conducted to assess the cost effectiveness of common interventions used to control Noncommunicable diseases in south Darfur. Noncommunicable diseases are becoming an increasing public health concern due to their economic significance, and their great impact on the ability of affected individuals to contribute to their communities, and the high burden it represents on the health system.

Objectives: To evaluate the role of health insurance fund in controlling Noncommunicable diseases. To assess cost effectiveness of the management of Noncommunicable disease within the National Health insurance fund system.

Methods: This study is designed to use secondary data from the national health insurance fund in south Darfur, comparing the cost for the management of 3 selected Noncommunicable diseases (Hypertension, Heart disease, and Diabetes). I have reviewed the entire data of the year 2017 and compared the cost effectiveness of the currently used interventions against health promotion and coordinated approach within the national health insurance fund.

Results: The 3 selected disease (Heart Disease, Hypertension and Diabetes) has significant burden on the health system, both financially and operationally. The NHIF concentrates on the management of Noncommunicable diseases without paying due attention to the importance of prevention strategies such as health promotion, community based integrated management of NCDs, and coordination among other sectors.

Conclusion: To effectively reduce the financial burden of Noncommunicable disease; the NHIF needs to concentrate on prevention of Noncommunicable diseases through extensive health promotion campaigns, promotion of healthy lifestyle, and coordinated approach to controlling Noncommunicable disease.
\end{abstract}

Keywords: Noncommunicable; Hypertension; Cardiovascular; Diabetes; Renal Disease

\section{Introduction}

Non-communicable diseases (NCDs) pose substantial health and economic challenges both at the individual and societal levels.Worldwide, noncommunicable diseases (NCDs) have emerged as the leading public health threat which accounts for the deaths of 38 million people annually [1]. Chronic non-communicable diseases (NCDs) affect more than $20 \%$ of the Indian population [2], with incidence and prevalence projected to increase substantially as the population aged 60 and over increases.

\footnotetext{
${ }^{*}$ Corresponding author: Ekram Adam Eldoom

Faculty of Public Health, University of Alzaeim Alazhari, Sudan.

Copyright (@ 2021 Author(s) retain the copyright of this article. This article is published under the terms of the Creative Commons Attribution Liscense 4.0.
} 
Total deaths from non-communicable diseases are projected to increase by a further $17 \%$ over the next 10 years The greatest increase will be seen in the African region (27\%) and the Eastern Mediterranean region (EMR=25\%). Therefore it is noticeable that the rapidly increasing incidence of these diseases is affecting poor and disadvantaged populations disproportionately, contributing to widening health gaps between and within countries. [3]

The main NCDs contributing to the burden of disease are;

- Cardiovascular Diseases and hypertension

- Diabetes Mellitus • Asthma

- Cancer

- Renal Disease

- General Injuries and road Traffic Accidents

- Mental Health Disorders

Sudan is the largest country in Africa. It has an area of 2.5 million $\mathrm{km} 2$. Decentralization was introduced as an appropriate system of governance compatible with the needs of the multi-ethnic and multi-cultural society of Sudan. The population of Sudan, comprising some 19 major ethnic groups, was over 39 million in 2009 census with $63.8 \%$ living in rural areas and the growth rate is $2.6 \%$, indicating that the population doubles every 27 years. Children of the age $0-14$ years comprise $42.6 \%$ of the total population while the under-five children comprise $14.9 \%$ of total population. The country is divided into 25 states and 134 Localities and the majority of the population is concentrated in six States of the Central Region with 60\% living around the River Nile [4].

The health system is founded upon a multi-tier government: federal, state and local governments. The federal level is concerned with policy making, planning, supervision \& co-ordination. The state governments are empowered to plan, policy making and implementation at state level. Some problems have appeared during the implementation of the federal system, the prominent problem is uneven distribution of financial resources \& manpower between states and between rural and urban areas. An unevenly distributed population is again demarcated by the fact that while the country shows a mean population density of 10 persons per square $\mathrm{Km}$ it reaches up to 50 at certain agricultural areas and in regions like Khartoum and Gezira and South Darfur it is five times as high as in the rest of the country. Natural disasters and civil conflict have also added to this uneven proportions through the high rates of rural-urban migration rates during the last two or more decades. Around 30\% of the population live in urban areas due to migration which includes large numbers of internally displaced persons (IDPs) mainly from southern Sudan but also from other parts of the country. The UN estimates that there are 4 million IDPs 800,000 of them are in south Darfur. In many cases, particularly in Khartoum, the distinction between IDPs and urban poor has become blurred over the years. Immigration of skilled professionals is also a significant issue in northern Sudan, causing a continuing brain drain Sudan is not an exception from the international trend of transition of disease pattern from predominantly communicable to noncommunicable diseases Sudan is part of EMR where currently $47 \%$ of the region's burden of disease is due to Noncommunicable diseases and it is expected that this will rise to $60 \%$ by the year 2020 [4].

Prevention and control of NCDs is one of the policy foundations of Sudan's National Strategic plan for the health sector (2003-2027) reflecting a strong political commitment towards NCDs. However, data on the disease burden of NCDs in Sudan is scarce and deficient. The situation is further complicated in south Darfur state by other factors in the country i.e. armed conflicts and scarce resources and poor capacity for future foreseeing and strategic planning. There is also lack of staff retention policies particularly at state and locality levels. Some fragmented efforts have been made at federal level to formulate control programs but these have not yet been materialized into tangible outcomes due to limited coverage of services, low effectiveness and absence of clear national policy to combat the diseases. Sudanese people are exposed all their lives to a range of risks to their health, whether in the shape of communicable or non-communicable disease, injury, consumer products, violence or natural catastrophe. Sometimes whole populations are in danger, at other times only an individual is involved before reviewing some of the available data an overview of Sudan is given first Non-communicable diseases (NCDs) are of increasing concern in low- and middle-income countries (LMICs). Humanitarian agencies and governments are increasingly challenged with how to effectively tackle NCDs. Reviewing the evidence of interventions for NCDs can help guide future policies and research by identifying effective interventions and evidence gaps. The aim of this paper is to systematically review evidence on the effectiveness of interventions targeting NCDs during and the cost effectiveness, comparing the cost between national health insurance fund and private clinic in south Darfur state [4]. 


\subsection{Burden of non-communicable disease globally}

Noncommunicable diseases (NCDs) are noninfectious health conditions typically caused by genetic and/or environmental and lifestyle factors. As NCDs tend to be long-lasting or recurrent, they are sometimes also referred to as chronic diseases [5]. Currently, NCDs account for almost two-thirds of deaths globally. Four main diseases account for the majority of deaths from NCDs: cardiovascular disease, chronic respiratory disease, cancer and diabetes. Importantly, these four NCDs share common behavioral risk factors including tobacco use, unhealthy diet, physical inactivity and harmful use of alcohol. Sudan does not have a surveillance system for NCDs and NCD risk factors but has only a few scattered studies [6, 7]. This is due to difficulties in the health information system and lack of research resulting from shortage of human and financial resources. The data to be presented here is from the NHIF. As per the health statistic records of the Federal Ministry of Health[8] Sudan Household Survey 2009 and the WHO STEPs study done in Khartoum there is a rising prevalence and an overwhelming impact of NCDs on the Sudan health system. From the data available the most common diseases in Sudan include diabetes mellitus, asthma, hypertension, ischemic heart disease, cancers, renal disease, general injuries and road traffic accidents, injuries and mental health disorders. These selected diseases accounted for $41 \%$ of all deaths in 2005 . This was 143,000 from 346,000 total deaths. Perhaps the most useful study to shed some light on the prevalence of NCDs and their risk factors was a WHO STEP wise risk factor indicator survey that was conducted in 2005 in Khartoum State, Sudan [4].

\subsection{Global response to NCDs}

In 2011, the World Health Organization (WHO) published a prioritized research agenda for the prevention and control of Noncommunicable diseases, which identified key areas of research relating to the prevention and control of NCDs. In September of that year, participants at the United Nations High-level Meeting on Noncommunicable Disease Prevention and Control recognized the existence of cost-effective interventions regarding the preventability of NCDs and the numerous opportunities for global action, and subsequently The Sixty-sixth World Health Assembly endorsed the Global Action Plan for the prevention and control of Noncommunicable diseases 2013-2020. This called upon international partners to take coordinated action to attain nine voluntary global NCD targets by 2025 - including a $25 \%$ reduction in premature mortality due to NCDs [6]. Noncommunicable diseases (NCDs), such as cardiovascular diseases, cancer, diabetes and chronic respiratory diseases, are the leading global cause of death and are responsible for $70 \%$ of deaths worldwide. These NCDs share key modifiable behavioral risk factors like tobacco use, unhealthy diet, lack of physical activity, and the harmful use of alcohol, which in turn lead to overweight and obesity, raised blood pressure, and raised cholesterol, and ultimately disease. They continue to be an important public health challenge in all countries, including low- and middle-income countries where more than three quarters of NCD deaths occur. Effectively tackling NCDs and their key risk factors requires a detailed understanding of the current status and progress being made at the country level. Feasible and cost-effective interventions exist to reduce the burden and impact of NCDs now and in the future. Tracking national implementation of a key set of tracer actions linked to these interventions allows for global benchmarking and monitoring of progress being made against NCDs. It also serves to highlight challenges and areas requiring further attention [6].

\subsection{Risk factors}

\subsubsection{Modifiable behavioral risk factors}

- Modifiable behaviors, such as tobacco use, physical inactivity, unhealthy diet and the harmful use of alcohol, all increase the risk of NCDs.

- Tobacco accounts for 7.2 million deaths every year (including from the effects of exposure to second-hand smoke), and is projected to increase markedly over the coming years.

- $\quad 4.1$ million annual deaths have been attributed to excess salt/sodium intake.

- More than half of the 3.3 million annual deaths attributable to alcohol use are from NCDs, including cancer.

- 1.6 million deaths annually can be attributed to insufficient physical activity.

\subsubsection{Metabolic risk factors}

- Metabolic risk factors contribute to four key metabolic changes that increase the risk of NCDs:

Raised blood pressure

Overweight/obesity

Hyperglycaemia (high blood glucose levels) and hyperlipidemia (high levels of fat in the blood).

- In terms of attributable deaths, the leading metabolic risk factor globally is elevated blood pressure (to which $19 \%$ of global deaths are attributed), followed by overweight and obesity and raised blood glucose [6]. 


\subsection{Management of Risk Factors}

The most common causes of NCDs are metabolic and behavioural risk factors and can be largely preventable by several available means. Most global discussions concern the risk factors of self-management (tobacco and alcohol consumption, physical activity, weight, food, and dental health care) and focus on the role of individual responsibility to manage the risk factors of NCDs. Health care specialists should educate patients about their nutrition value and raise the profile of didactics, practicums, and workshops in daily practice [9]. Interventions are used in public health management in an effort to promote good health behaviour. Researchers also highlight the environmental factors (air pollution, climate changes, sunlight) and their impact on NCD development. Air pollution will be an important challenge in the future and new technologies, such as microchips, will have more of an impact in air monitoring [10].

\section{Methodology}

This study is designed to use secondary data from the national health insurance fund in south Darfur, comparing the cost for the management of 3 selected Noncommunicable diseases (Hypertension, Heart disease, and Diabetes). I have reviewed the entire data of the year 2017 and compared the cost effectiveness of the currently used interventions against health promotion and coordinated approach within the national health insurance fund.

\section{Results}

- From the data shown in table (1) from the NHIF in south Darfur the total financial cost for the management of 3 selected Noncommunicable disease (Heart disease, Hypertension, and Diabetes) was 944,541SDG(33,000 USD) in 2017, and for all Noncommunicable disease it was3,588,780 SDG, 123,751 USD. This large amount of budget can be reduced to less than $50 \%$ if we followed more effective strategies such as mass health promotion, abandonment of tobacco, regular exercise, etc.

- If we assumed that average years per patient is 10 years, then the average amount of money required to be invested to treat a patient with hypertension will be 662 SDG, and for the 12996 patient 8,602,105 SDGs, and for a patient with heart disease the cost per 10 years is around 1930 SDG, and for the 390 patient 752930 SDGs.

- Its known that NHIF is the cheapest health service provider in the country, where only $25 \%$ of the total cost is paid by the patient, with that assumption we can estimate the total cost for Noncommunicable diseases in private sector to be around 3,778,164 SDG, 130,281 USD per year, an amount that can support effective community based interventions for NCDs, including extensive and sustainable health promotion.

- The NHIF system for the management of NCDs doesn't integrate health education and promotion programs targeting patients coming for services.

- The information system at NHIF in south Darfur showed some inconsistency in terms of average cost per patient, as shown in figures 1, 2 and 3, this can be due to problems with the information system itself where the denominator is not constant, or due to different services being provided to different patient every visit.

- $\quad$ From the revision of the data it was observed that the majority of patients visiting the NHIF centers in south Darfur were patients with hypertension, around 12905 (22.80\%), followed by heart disease 390 $(0.70 \%)$, and diabetes $170(0.30 \%)$.

- $\quad$ NHIF in south Darfur is not represented in the existing coordination forum for health, therefore there is no coordinated approach to controlling NCDs.

- $\quad$ The NCD with the highest cost for treatment is heart disease with maximum of 193 SDG per patient per visit, while Diabetes showed the lowest average cost with 35 SDG per patient per visit table 1.

Table 1 Total number of cases with average cost per patient in 2017.

\begin{tabular}{|l|l|l|l|}
\hline Disease & Frequency & Total cost & Average cost \\
\hline Heart disease & 390 & 75293 & 193 \\
\hline Hypertension & 12996 & 860211 & 66 \\
\hline Diabetes & 170 & 9037 & 53 \\
\hline Total & 13556 & 944541 & 312 \\
\hline
\end{tabular}




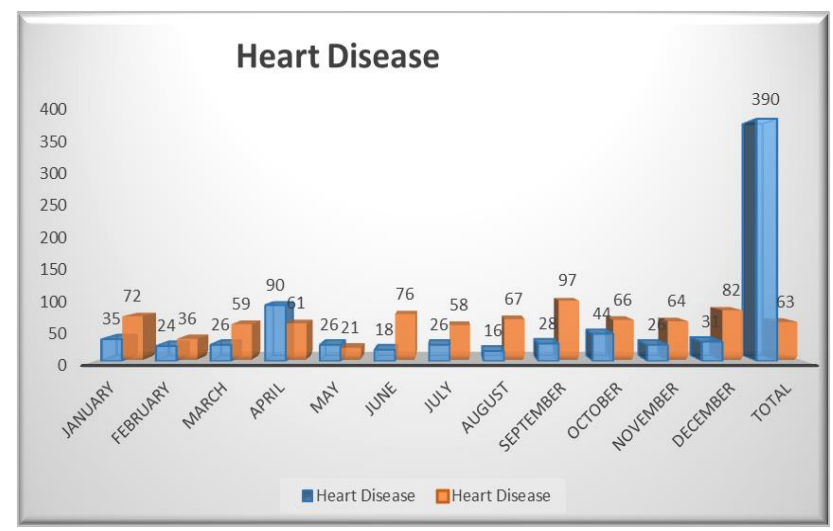

Figure 1 Shows the frequency and average of heart disease cost per Month in 2017.

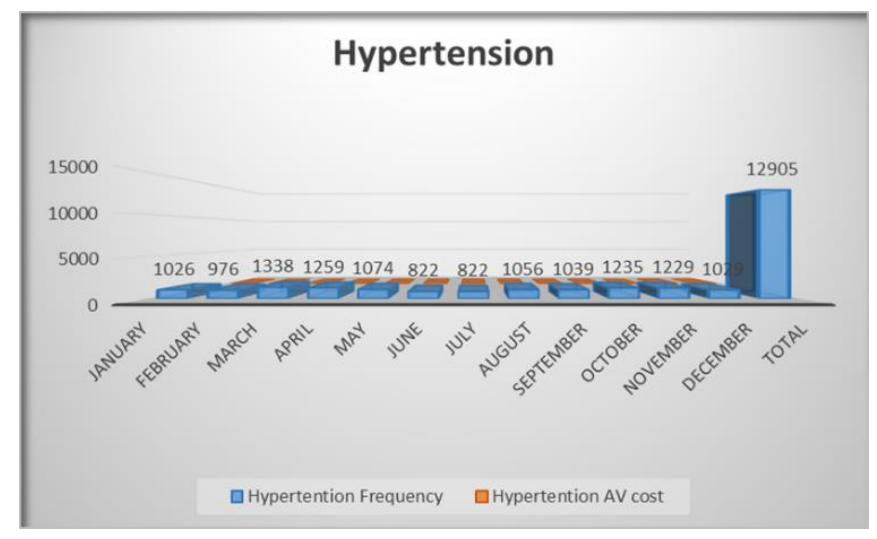

Figure 2 (Frequency and average cost for management of hypertension in 2017)

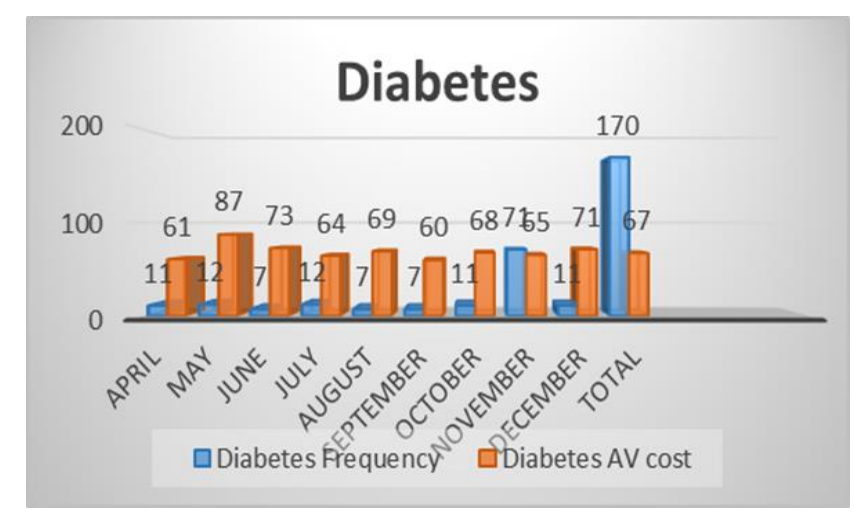

Figure 3 Frequency and average cost for Diabetes in 2017

\section{Discussion}

\subsection{Prioritize health promotion and disease prevention}

In order to reduce the burden of NCDs on the health system and individual levels, our health system needs to focus on health promotion and prevention of risk factors. A significant proportion of the NCD burden can be prevented, and health promotion and risk reduction must be prioritized for people at all stages of these diseases-people without disease, at risk of disease, and with chronic disease of varying levels of complexity. Health promotion is the process of enabling people to increase control over and improve their health. Interventions must include actions not only at the 
level of the individual, but also aimed at building healthy public policy, creating supportive environments, strengthening community action, developing personal skills and reorienting health services[10].

Prevention and risk reduction strategies acknowledge that there are known preventable risk factors, such as smoking and lack of physical activity, that have impact on the development and progression of many of the chronic diseases. These risk factors should be the focus of significant, targeted and coordinated action. Using the emerging evidence, appropriate and effective prevention interventions must be identified, implemented and sustained by engaging not only the health sector in health promotion and illness prevention, but also reaching beyond the health sector to all those sectors and settings that impact on the risk and protective factors for non-communicable disease[6].

\subsection{Facilitate coordinated and integrated multidisciplinary care across services, settings and sectors to develop strong process to assist in mobilizing resources}

Influencing the changes in polices in other non-health sectors like trade, education, agriculture, urban development, food and pharmaceutical production and Integration of prevention and control of NCDs into the national health development plan will improve the care of people with chronic disease. This coordination also includes general practice, community health facilities, private providers, and community and non-government organizations. It may also require community and disability support, as well as support from family and care providers.

The integrated provision of NCD prevention and care requires a flexible health system that can coordinate care planning across services, settings, sectors and over time. This means commitment from a range of services and sectors, and the ability to work together to achieve shared goals. Multidisciplinary care planning must be person centered, incorporate prevention, self-management and co-morbid conditions, and be responsive to changing patient needs.

In 2017 around 380 patients with heart diseases were seen in all NHIF centers in south Darfur, total cost for the 390 patient is around 75293 SDG, with average cost per patient of 193 SDG. According to health care providers within NHIF most of the patient were provided with education on exercise and dietary control, but the health education exercise is not part of the agreed protocol.

It's also clear that the existing coordination forum regarding health is totally dedicated to tackle communicable diseases, there is a dare need to incorporate NCDs in the existing health coordination forum to improve availability of resources, integrated approach to management and control of NCDs, and creating opportunities for fund raising for NCDs together with communicable diseases.

\section{Conclusion}

- To effectively reduce the financial burden of Noncommunicable disease; the NHIF needs to concentrate on prevention of Noncommunicable diseases through extensive health promotion campaigns, promotion of healthy lifestyle, and coordinated approach to controlling Noncommunicable disease.

- Research is important, not only for the development of new affordable and effective diagnostics, treatment and care of NCDs, but also as a crucial tool to display cases of evidence-based approaches that ultimately bring about healthy behaviour changes.

- Advocate for inclusion of NCDs in the discussion agenda during existing (health coordination meeting, Nutrition cluster meeting, and SRH sub-sector meetings).

- Prevention of NCDs is way better and effective than managing in reducing the burden on the health system in south Darfur state.

- NHIF and private sectors has to play a greater role in the control of NCDs through regular community mobilization, awareness, and health promotion.

- The NHIF in south Darfur shall review their information system to be more informative and consistent, as it's realized that there are inconsistencies in the average cost for each disease.

\section{Compliance with ethical standards}

\section{Acknowledgments}

We are grateful to all of those with whom we have had the pleasure to work during this and other related projects. Each of the members of our faculty Committee has provided us extensive personal and professional guidance and taught us a great deal about both scientific research and life in general. 


\section{Disclosure of conflict of interest:}

Yousif Adam Hussien Noreldin, and Ekram Adam Eldoom declare that they have no conflict of interest.

\section{References}

[1] World Health Organization. Non-Communicable Diseases - Fact Sheet No. 355. 2015; Int J Prev Med. 2016; 7: 54.

[2] Patel V, Chatterji S, Chisholm D, Ebrahim S, Gopalakrishna G, Mathers C, Mohan V, Prabhakaran D, Ravindran RD, Reddy KS. Chronic diseases and injuries in India. Lancet. 2011; 377(9763): 413-28.

[3] Global Report. Preventing Chronic Diseases a vital investment. 2005.

[4] Republic of the Sudan National Ministry of Health Directorate General of Public health and Emergency. Noncommunicable Disease National Strategic Plan 2010-2015.

[5] WHO. Noncommunicable diseases. 2018.

[6] World Health Organization. Global status report on noncommunicable diseases. Geneva: WHO. 2014.

[7] WHO. Noncommunicable diseases global monitoring framework: indicator definitions and specifications. Geneva: WHO. 2014.

[8] Global Health Data Exchange. Sudan - Khartoum STEPS Noncommunicable Disease Risk Factors Survey. 20052006.

[9] Johnston E, Mathews T, Aspry K, Aggarwal M, Gianos E. Strategies to fill the gaps in nutrition education for health professionals through continuing medical education. Curr Atheroscler Rep. 2019; 21: 13.

[10] Schultze F, Gao X, Virzonis D, Damiati S, Schneider MR, Kodzius R. Air quality effects on human health and approaches for its assessment through microfluidic chips. Genes. 2017; 8: 244. 